\title{
User interaction and workflow management in Grid enabled e-VLBI experiments
}

\section{Dominik Stokłosa ${ }^{1}$}

Poznań Supercomputing and Networking Center Noskowskiego 10 street, 61-704 Poznań, Poland

E-mail:d.stoklosa@man.poznan.pl

\section{Norbert Meyer}

Poznań Supercomputing and Networking Center Noskowskiego 10 street, 61-704 Poznań, Poland E-mail: meyer@man.poznan.pl

The purpose of this paper is to present a design of the integration of radio-astronomical VLBI experiments with the Grid environment. The current status of the VLBI process is described, and the main part of this document presents the proposed architecture for the development of next generation e-VLBI systems. This design was created for the purpose of EXPReS project [3], partially funded by the European Commission (FP6 - IST).

Science and Technology of Long Baseline Real-Time Interferometry: The 8th International e-VLBI Workshop

Madrid, Spain

June 22-26, 2009

\footnotetext{
$1 \quad$ Speaker
} 


\section{Introduction}

Since 1970s telescopes from all over the world have been combined to perform Very Long Baseline Interferometry. Data received at each antenna is paired with timing information, usually from a local atomic clock, and then stored for later analysis on a magnetic tape or hard disk. Later, the data is correlated with similarly recorded data from other antennas to produce a resulting image. Resolution of images depends on the overall size of the network and the sensitivity depends on a total collecting area of all telescopes involved. After data from all telescopes is collected, it must be put together and correlated in order to produce final observation result. In Europe it is done by the European VLBI Network [2] formed by a consortium of five major radio astronomy institutes in Europe.

A decade ago, a 20.000 node PC cluster would have been required to match the processing power of the EVN MkIV Data Processor at JIVE [1]. However, this number has been falling rapidly as processor capabilities continue to develop. The networking infrastructure is also getting more capable of dynamically handling very large data transfers in long periods of time. This has led to introduction of e-VLBI System, with the main difference being data sent via network to the central processing facility at JIVE. This has drastically shortened the time required to obtain final results from actual observation. However, in e-VLBI approach a new bottleneck has emerged - the single central data processor at JIVE. A concept of software-based distributed correlation embedded in a Grid Computing environment has been introduced to solve this problem [5]. 


\section{The e-VLBI System}

The e-VLBI System has been constructed to provide tools for managing the distributed software correlation. The system consists of the following components: Workflow Manager (WFM), Translation Node (TN), VLBI Broker (VB), Correlation Node (CN) and Correlated Data Service (CDS). The interaction between components is presented in the Figure 1. The detailed description of each system component is presented further in this chapter.

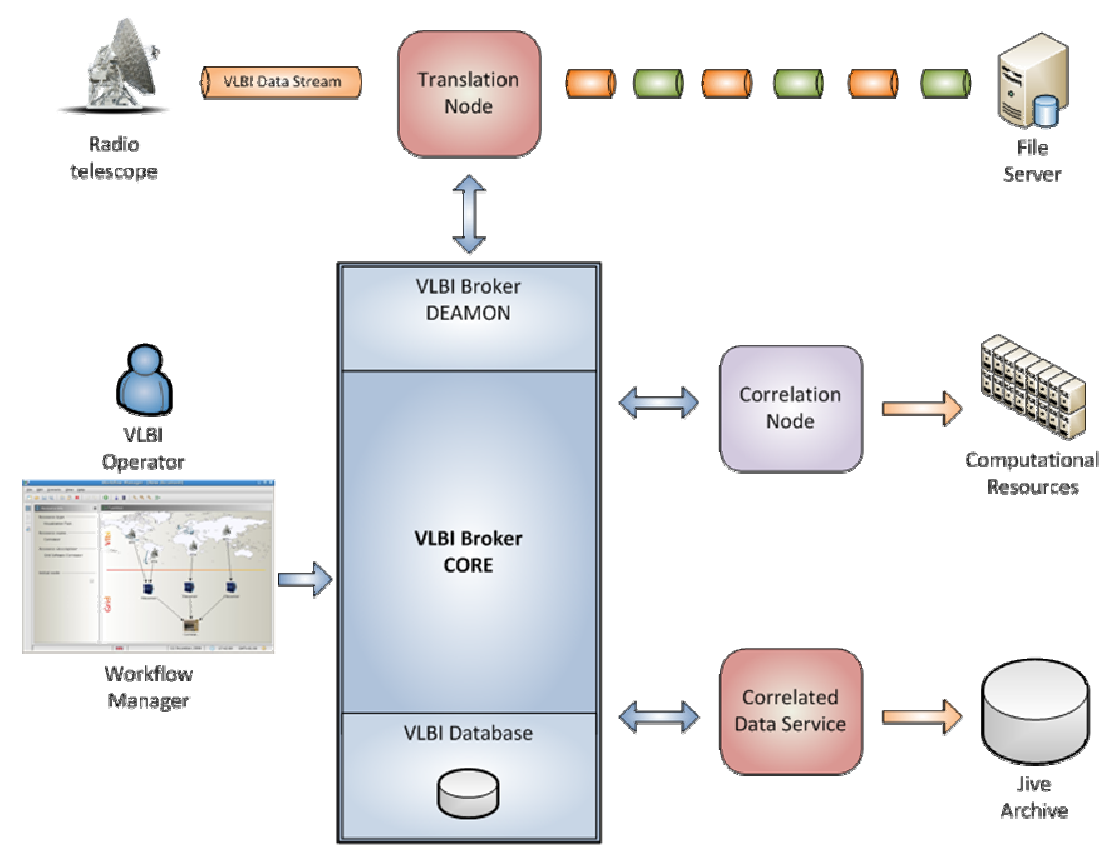

Figure 1 The general architecture of e-VLBI System

E-VLBI System allows astronomers to plan, execute and monitor their observations in the form of so called workflows. However, the e-VLBI experiment is not only the observation itself. The e-VLBI experiment consists of a definition of storage elements, a definition of data flows or a definition of computation resources, etc. Such an e-VLBI workflow has to be created for each observation. The Workflow Manager Application (WFM) has been created to allow users to design and execute their observation workflows easily.

VLBI Broker (VB) is the central element of the e-VLBI System providing the centralized control of the entire experiment. The VB module processes experiment definition submitted from the WFM application and forwards tasks' description to telescope sites (Translation Nodes) and correlation sites (Correlation Nodes). This module is also responsible for coordination and submission of computational jobs with the distributed correlation of chunked data.

Translation Node (TN) responsible for handling data from radio telescopes and preparing data for correlation. The data stream from radio telescope is buffered and divided into a smaller chunks. Chunk sets (every corresponding chunk from each data source) are temporarily stored 
within File Server disk space until they are correlated. There can be many TNs involved in VLBI experiment. However, it is required that each radio telescope has one Translation Node assigned. The TN module is informed about each new experiment by VLBI Broker.

Correlation Node $(\mathrm{CN})$ - responsible for managing and executing correlation jobs on the cluster/grid.

Correlated Data Service (CDS) - service responsible for storing correlated data in the Jive Archive.

\section{How to conduct a VLBI experiment with the e-VLBI System}

This chapter presents in detail how to conduct an experiment using e-VLBI System. This process has been divided into four phases: creating an observation workflow, submitting workflow for execution, workflow execution (the actual correlation) and finally monitoring the correlation process. Each phase is described below.

1. Creating an observation workflow using a WFM application

An e-VLBI user creates an experiment description (VEX file) using SCHED application. This process is no different than in regular VLBI observations. The VEX is processed in the Workflow Manager Application (WFM) by an e-VLBI operator. Experiment control parameters can be verified and modified if necessary. A central eVLBI operator will be able to associate file servers with radio telescopes. This tells the VLBI System where to stream data from radio telescopes. The last step is adding a computational resources which will be used to correlate data. Each computational resource represents a separate, independent distributed software correlator module which can be used to correlate data chunks.

2. Submitting the observation workflow for execution in the Grid environment

A completed observation workflow with file servers defined and assigned to radio telescopes, with correlation nodes defined and other correlation parameters set up can be submitted for execution using the Workflow Manager Application. The next step actual data chunks correlation is done behind the scene by the e-VLBI System.

\section{The workflow execution}

The observation workflow, together with observation parameters is sent to the VLBI Broker. The broker module informs all Translation Nodes specified in the observation workflow about each new experiment. Each $\mathrm{TN}$ is responsible for preparing the environment for retrieving data from data source and dividing it to smaller chunks. In the mean time VB informs also each Correlation Node about each new experiment. Each CN prepares environment for chunks correlation. The process of delay table generation is scheduled by $\mathrm{CN}$, before first chunks are ready for correlation. This happens only once, at the beginning of the correlation process.

Whenever each new data chunk is ready for correlation TN module informs the VLBI Broker about its location. The VB broker stores information about chunk locations from all data sources. When a complete data set, within the same time boundaries is collected, it is submitted for correlation. The VB module contacts one of 
the Correlation Nodes, which handles the correlation process. The decision on which Correlation Node should be used is taken based on internal algorithm. The actual load

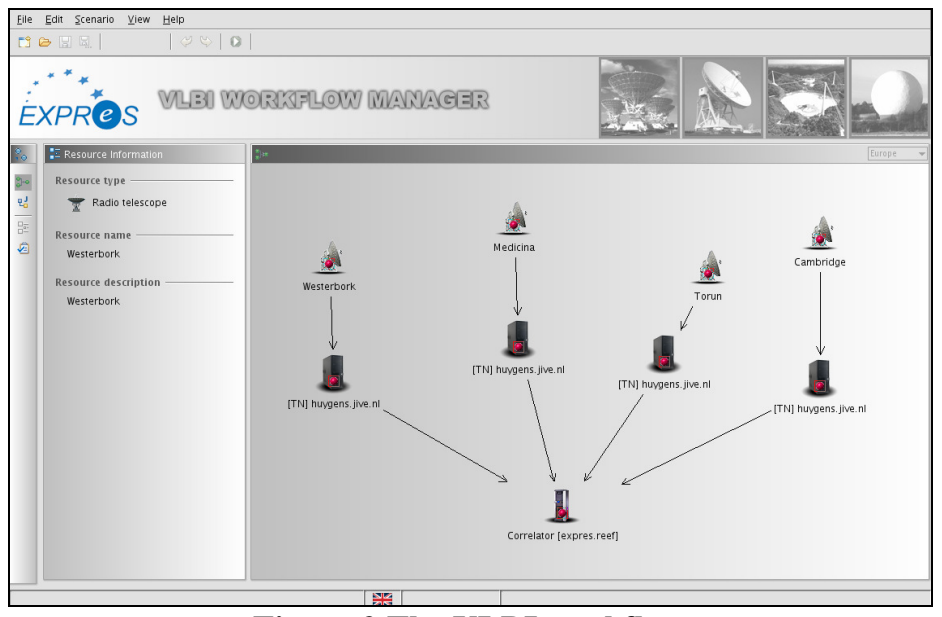

Figure 2 The VLBI workflow

of each $\mathrm{CN}$ is taken into consideration. $\mathrm{CN}$ generates a special script which starts software correlator. Please note that a number of $\mathrm{CN}$ is configurable and is set up by VLBI operator during stage 1: workflow creation. The status of the correlation job is monitored by $\mathrm{CN}$ module and propagated back to the VB module. The status can be displayed in the Workflow Manager Application. When a data set has been correlated, $\mathrm{CN}$ sends a notification to the VB module with a result location. The VB informs the Correlated Data Service that new data chunk has been correlated. The CDS service is responsible for storing this correlated data product in the Jive archive. When correlated data is stored in the database it is removed, together with data chunks from the computation resources. This process is repeated until all data chunks are correlated.

\section{Monitoring the VLBI experiment}

As it was mentioned before the correlation progress can be viewed in the Workflow Manager Application. It is possible to see the total number of data chunks, number of correlated data chunks and finally the number of data chunks, which still need to be correlated.

\section{Summary}

The paper describes a possible approach to conduct a VLBI experiments using distributed software correlator deployed on the Grid. The e-VLBI System has been presented in detail and finally it was shown how to conduct e-VLBI experiment with e-VLBI System.

One of the objectives of EXPReS project was to show that distributed software correlation is possible. Sample experiments have been conducted with a distributed software correlation modules running on two separate clusters at PSNC [4] (Poznan, Poland). Data has been served by Translation Node deployed at Jive [1] (Dwingeloo, Netherlands).

The prototype system which has been constructed proved that distributed, software correlation with Grid resources is possible and can be used in the future experiments. 


\section{Acknowledgements}

The work presented in this paper was carried out with funding from European Commission under EXPReS project (6th Framework Programme - IST), contract number 026642.

\section{References}

[1] JIVE website - http://jive.nl

[2] EVN website - http://evlbi.org

[3] EXPReS website - http://www.expres-eu.org/

[4] PSNC website, http://www.man.poznan.pl/pcss/public/main/index.html?lang=en

[5] Okon, M.; Stoklosa, D.; Oerlemans, R.; Langevelde, H.J. van; Kaliszan, D.; Lawenda, M.; Rajtar, T.; Meyer, N.; and Stroinski, M. "Grid Integration of Future Arrays of Broadband Radio-Telescopes moving towards e-VLBI." Grid Enabled Remote Instrumentation. Springer, 2008. p. 571. 